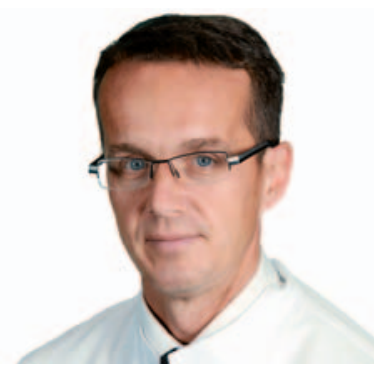

\title{
COPD- und ILD-Patienten profitieren von pneumologischer Rehabilitation nach Lungentransplantation
}

\section{Marc Spielmanns}

Zürcher RehaZentrum Wald, Wald, Schweiz

Abstract aus Schneeberger T, Gloeckl R, Welte T, Kenn K: Pulmonary rehabilitation outcomes after single or double lung transplantation in patients with chronic obstructive pulmonary disease or interstitial lung disease. Respiration 2017; 94: 178-185.

\section{Schlüsselwörter}

Lung transplantation . Chronic obstructive pulmonary disease Interstitial lung disease · Pulmonary rehabilitation .

Exercise training

\section{Summary}

Background: Pulmonary rehabilitation (PR) following lung transplantation ( $(\mathrm{TX})$ has been shown to be effective with regard to exercise capacity and health-related quality of life (HRQL). However, outcome data is limited with respect to LTx as a population. Differences concerning the effects of PR in patients with single LTx (SLTx) or double LTx (DLTx) have not been studied yet. Objectives: The aim was to compare possible differences concerning PR outcomes between SLTX and DLTX.

Methods: In a retrospective analysis (period: 1997-2016), data from 722 patients with either chronic obstructive pulmonary disease (COPD; SLTx: $n=129$, FEV1 $51 \pm$ 17\% pred; DLTx: $n=204$, FEV1 $74 \pm 20 \%$ pred.) or interstitial lung disease (ILD; SLTx: $n=135$, FVC $58 \pm 18 \%$ pred.; DLTx: $n=254$, FVC $63 \pm 18 \%$ pred.) after LTx were included. All patients underwent a specialized inpatient PR program. The data of the 6-minute walk distance (6MWD) and HRQL (physical [PCS] and mental [MCS] component summary of the SF- 36 questionnaire) were analyzed. Results: Independently from the procedure and pretransplant diagnosis, patients significantly $(p<0.05)$ improved the 6MWD without any differences between SLTx and DLTx (COPD: SLTx: $+109 \pm 68$ m, DLTx: +117 \pm 82 m; ILD: SLTx: $+115 \pm 80$ m, DLTx: $+132 \pm 77$ m). The PCS (COPD: SLTx: $+9 \pm$ 9 points, DLTx: $+7 \pm 9$ points; ILD: SLTx: $+6 \pm 9$ points, DLTx: $+9 \pm$ 9 points) and MCS (COPD: SLTX: $+8 \pm 15$ points, DLTx: $+7 \pm 15$ points; ILD: SLTx: $+10 \pm 13$ points, DLTx: $+8 \pm 12$ points) also improved significantly without any group differences.

Conclusions: LTx patients with a pretransplant diagnosis of COPD or ILD all benefitted significantly and with clinical relevance with regard to exercise capacity and HRQL from an inpatient PR performed within 1 year postoperatively. PR outcomes were similar regardless of SLTX or DLTX.

(c) 2017 S. Karger AG, Basel

\section{KARGER}

(C) 2018 S. Karger GmbH, Freiburg 


\section{Transfer in die Praxis}

\section{Hintergrund}

Bei terminalen Lungenerkrankungen ist die Lungentransplantation (LTX) die Ultima Ratio. Weltweit wurden bislang über 40000 Lungentransplantationen durchgeführt. Neben Patienten mit chronisch obstruktiver Lungenerkrankung (COPD) zählen Patienten mit interstitieller Lungenerkrankung (ILD) zu den häufigsten Empfängern einer Spenderlunge. Die Tatsache, dass die LTx als Einzel-Lungentransplantation (SLTX) oder Doppel-Lungentransplantation (DLTx) vollzogen werden kann, macht die Operation einzigartig. In einem gemeinsamen Statement folgerten die American Thoracic Society und die European Respiratory Society, dass es bezüglich der natürlichen Überlegenheit der DLTx im Vergleich zur SLTx an Evidenz mangelt. Dies könnte neben dem Fehlen von randomisierten, kontrollierten Studien auch der Patienten-Heterogenität in bisherigen Studien geschuldet sein. Dadurch, dass randomisierte Studien zur Untersuchung eventueller Vorteile/Unterschiede von SLTX und DLTx unmöglich sind, stellen retrospektive Analysen von bestehenden LTx-Datenbanken eine wichtige Informationsquelle dar.

\section{Ergebnisse der Studie}

Erwartungsgemäß gab es zwischen SLTx und DLTx zum Zeitpunkt der Aufnahme der pneumologischen Rehabilitation (PR) signifikante Unterschiede hinsichtlich der Lungenfunktion. Dies hatte jedoch keinen Einfluss auf die körperliche Leistungsfähigkeit, da das Ausgangsniveau der 6-Minuten-Gehteststrecke zwischen den Gruppen vergleichbar war. Wenn man die Ergebnisse zusammenfasst, lässt sich sagen, dass COPD- und ILD- Patienten nach der LTx $(\leq 1$ Jahr) in signifikantem und klinisch relevantem Ausmaß von einem PR-Programm profitierten. Hinsichtlich der Verbesserungen der körperlichen Leistungsfähigkeit und der gesundheitsbezogenen Lebensqualität gab es keine signifikanten Unterschiede zwischen SLTX und DLTX. Es scheint daher, dass SLTx und DLTX-Patienten vergleichbar gut trainierbar sind und beide Gruppen im selben Ausmaß von einer PR profitieren. Auch die innerhalb der PR aufgetretenen Komplikationsraten von Zytomegalievirus (CMV)- und bakteriellen Infektionen war zwischen den beiden LTx-Typen vergleichbar.

\section{Fazit und Transfer in die Praxis}

Nach einer LTx treten trotz annähernd normalen Lungenfunktions- und Gasaustauschwerten oftmals erhebliche Einschränkun- gen der körperlichen Leistungsfähigkeit, Aktivität sowie der Lebensqualität auf, die oft sogar noch Jahre nach der Transplantation und unabhängig des angewandten Verfahrens (SLTX/DLTX) bestehen [1]. Folglich empfinden LTx-Patienten die muskuläre Ermüdung bei Belastungen im Vergleich zu den Einschränkungen bei der Atmung eher als leistungslimitierend. Strukturelle Veränderungen wie z.B. reduzierte Muskelmasse, geringerer Anteil an Typ1-Muskelfasern sowie verminderte Aktivität oxydativer Enzyme begünstigen einen anaeroben Stoffwechsel der peripheren Muskulatur ähnlich dem von Patienten mit fortgeschrittener COPD [1]. Darüber hinaus können auch postoperative Aspekte wie z.B. eine verlängerte Liegezeit im Krankenhaus oder Nebenwirkungen der Immunsuppressiva einen negativen Einfluss auf die Muskelfunktion haben [2]. Um dem entgegenzuwirken, können daher Patienten auch nach einer LTx eine Indikation für eine PR darstellen. Die Studie zeigt, dass PR einen klinisch relevanten Zugewinn der körperlichen Leistungsfähigkeit von LTx-Empfängern erzielen kann und sich dies auch in der Lebensqualität wiederspiegelt. Vielmehr scheint es aber auch, dass COPD- oder ILD-Patienten nach SLTX und DLTX, zumindest im ersten Jahr nach der LTx, gleichermaßen trainierbar sind und von einer komplexen PR körperlich und psychisch vergleichbar gut profitieren. Die Frage nach der Nachhaltigkeit dieses Reha-Effektes und dessen Einfluss auf die Prognose der LTx-Empfänger bleibt offen.

\section{Disclosure Statement}

Hiermit erkläre ich, dass keine Interessenskonflikte in Bezug auf den vorliegenden Kommentar bestehen.

\section{Literatur}

1 Langer D: Rehabilitation in patients before and after lung transplantation. Respiration 2015;89:353-362

2 Maury G, Langer D, Verleden G, et al.: Skeletal muscle force and functional exercise tolerance before and after lung transplantation: a cohort study. Am J Transplant 2008;8:1275-1281.

Kontaktadresse: Dr. med. Marc Spielmanns, Zürcher RehaZentrum Wald, Faligbergstrasse 7, 8636 Wald, Schweiz, marc.spielmanns@zhreha.ch 